\title{
Does Internal Mammary Node Irradiation for Breast Cancer Make a Significant Difference to the Diameter of the Internal Mammary Artery? Correlation with Computed Tomography
}

\author{
Gamze Durhan $^{a}$ Ahmet Gürkan Erdemir ${ }^{a}$ Sezin Yuce Sari ${ }^{b}$ Melis Gultekin ${ }^{b}$ \\ Jale Karakayac Meltem Gülsün Akpınar ${ }^{a}$ Gökhan Özyiğit ${ }^{b}$ Ferah Yildiz ${ }^{b}$ \\ Figen Demirkazık ${ }^{\mathrm{a}}$

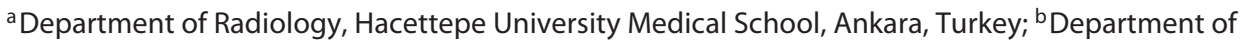 \\ Radiation Oncology, Hacettepe University Medical School, Ankara, Turkey; ${ }^{\mathrm{C}}$ Department of Biostatistics, \\ Hacettepe University Medical School, Ankara, Turkey
}

\author{
Keywords \\ Radiation therapy · Internal mammary artery · Computed \\ tomography
}

\begin{abstract}
Objective: Lymphatic irradiation in breast cancer improves locoregional control and has been shown to decrease distant metastasis. However, irradiation also accelerates the formation of atherosclerosis and can cause stenosis, not only in the coronary arteries but also in the internal mammary artery (IMA). The aim of this study was to investigate the effects of radiation on IMAs via computed tomography (CT). Methods: We reviewed the data of 3,612 patients with breast cancer treated with radiotherapy (RT) between January 2010 and December 2016. We included 239 patients with appropriate imaging and nodal irradiation in the study. All patients were treated with lymphatic irradiation of 46-50 Gy, and a boost dose (6-10 Gy) to the involved internal mammary nodes (IMNs) when imaging studies demonstrated pathological enlargement. Bilateral IMA diameter and the presence of calcification were assessed via thin contrast-enhanced CT and those of ipsilateral irradiated IMAs were compared with those of contralateral nonirradiated IMAs.
\end{abstract}

Results: The mean diameter of irradiated IMAs was significantly shorter than that of nonirradiated IMAs, regardless of laterality. All vascular calcifications were determined on the irradiated side. A boost dose of radiation to the IMNs and radiation technique did not significantly affect the IMA diameter or the presence of calcification. Conclusions: The diameter of the IMA is decreased due to RT regardless of laterality, radiation technique, and administration of a boost dose. Evaluation of vessels on $\mathrm{CT}$ before coronary artery bypass graft or flap reconstruction can help the surgeon select the most appropriate vessel.

(c) 2020 S. Karger AG, Basel

\section{Introduction}

The internal mammary lymphatic chain is one of the 3 lymphatic drainage stations of the breast in addition to the axilla and supraclavicular fossa (SCF). Previous studies have shown that the incidence of metastasis to internal mammary nodes (IMNs) in breast cancer is 4-9\% in patients with negative axillary lymph nodes (LNs) and 16$65 \%$ in patients with positive axillary LNs, respectively [1, 2]. In patients with LN-positive or high-risk LN-negative 
breast cancer, irradiation of the IMN and medial supraclavicular LNs improves the rates of disease-free survival (DFS) and distant metastasis-free survival (DMFS), and reduces the rate of breast cancer mortality compared to whole breast irradiation alone [3, 4]. However, radiotherapy (RT) to regional lymphatics, particularly IMNs, may cause long-term toxicity due to the close proximity to the heart and vascular structures. Accelerating atherosclerosis is one of these toxicities, and it is caused by vascular endothelial damage and increased inflammation $[5,6]$. Irradiation of the IMNs can cause stenosis, not only of the coronary arteries but also the internal mammary artery (IMA) which is located next to the IMNs.

Two aspects of the IMA are of major importance in female patients diagnosed with breast cancer. First, it is an excellent conduit for coronary bypass grafting (CABG), and its patency is extremely important for breast cancer patients as they are more likely than the general population to develop heart disease [7-9]. Second, the IMA is the most common recipient vessel used in free-flap breast reconstruction in breast cancer patients who have a mastectomy. The advantages of IMA for this are its sufficient length, wide diameter, high negative pressure, size match with deep inferior epigastric vessels, and central location for both microanastomosis and flap positioning [10]. Moreover, utilization of the IMA protects the thoracodorsal vessels and saves the latissimus dorsi flap as a "lifeboat" for salvage reconstruction in the case of freeflap failure [10-13]. In the light of these data, the aim of this study was to investigate the effect of RT on IMAs.

\section{Materials and Methods}

\section{Patients}

The data of 3,612 patients with breast cancer treated with RT between January 2010 and December 2016 were reviewed. We reevaluated the thorax CT scans performed on patients with abnormal chest radiograph, dyspnea symptoms, and suspicious findings on initial staging CT (for locally advanced breast cancer) until November 2018. Finally, 239 patients met the following inclusion criteria: unilateral breast cancer treated with surgery followed by RT to the chest wall/whole breast and regional lymphatics including IMNs, the SCF, and the axilla; follow-up contrast-enhanced thorax $\mathrm{CT}>1$ year after RT; and adequate CT image quality for evaluation of the IMA. The nonirradiated side served as the control for each patient. Exclusion criteria were male gender, bilateral breast cancer, chest irradiation for reasons other than breast cancer, receiving breast or chest wall RT alone, no available follow-up thorax CT after RT, follow-up thorax CT performed $<1$ year after RT, and inadequate CT images for evaluation of the IMA. The flow chart of how cases were selected is shown in Figure 1.

\section{Radiotherapy}

Prior to RT, a planning CT had been performed with a slice thickness of $2.5-5 \mathrm{~mm}$ with the patient in a supine position with a breast-board, starting from the temporomandibular joints to the level of the second lumbar vertebrae. RT had been performed via 3-dimensional conformal RT (3D-CRT), forward-planned intensity-modulated RT (FP-IMRT) or inverse-planned (IP)-IMRT. The irradiated volume had been the breast or chest wall and regional nodal lymphatics, encompassing the ipsilateral axilla (level 3 or full axilla, depending on the adequacy of the dissection and presence of an extracapsular extension), SCF, and IMNs. All target volumes had been contoured according to the Radiation Therapy Oncology Group (RTOG) guideline [14]. Based on this guideline, IMNs in the first 3 intercostal spaces had been delineated. The indications for IMN irradiation were radiologically or pathologically positive IMNs detected at the time of diagnosis or during treatment, inner quadrant tumors, or heavy metastatic axilla (a metastatic/dissected axillary LN ratio $>50 \%$ ) before 2015, and in all patients with positive axillary LNs after 2015 as long as the ipsilateral lung and heart doses had remained within the tolerance limits.

The prescribed total RT dose had been 50 Gy in 2-Gy daily fractions. In patients with positive IMNs at the time of diagnosis, a boost dose of 6-10 Gy was also administered to the intercostal area containing the metastatic LNs. Planning target volumes (PTV) was defined as clinical target volume (CTV) plus a margin of $0.5-1 \mathrm{~cm}$ for 3D-CRT. For the IMRT plans, PTV had been formed by adding $7 \mathrm{~mm}$ to the chest wall or breast CTV, and $5 \mathrm{~mm}$ to the axilla, SCF, and IM CTVs. Organs at risk had also been contoured at each slice. Treatment plans had been optimized according to the requirement that $\geq 95 \%$ of the PTV and $\geq 99 \%$ of the CTV had received $95 \%$ of the prescribed dose. RT was administered using the Philips SL25 (Philips Healthcare, Andover, MA, USA), the Elekta Synergy Platform (Elekta AB, Stockholm, Sweden), or the Varian Clinac DHX high-performance (Varian Medical Systems, Inc. Palo Alto, CA, USA) treatment machines.

\section{Imaging}

We evaluated the diameter of IMAs with only 1 follow-up CT obtained at least 1 year after RT. Bilateral IMA diameter and the presence of calcification were assessed using thorax CT (GE Lightspeed 16 Slice CT Scanner, New York City, NY, USA). After injecting an iodinated contrast agent $(300 \mathrm{mg} / 100 \mathrm{~mL}$ Iohexol) into the antecubital vein via a 21-gauge catheter at a rate of $2.5 \mathrm{~mL} / \mathrm{s}$ using a dual-chamber power injector, $30 \mathrm{~mL}$ of saline was infused in all patients. The CT imaging parameters were: 1.25 -mm slice thickness, a gantry rotation time of $0.6 \mathrm{~s}$ (pitch 1.375), a tube voltage of $120 \mathrm{kV}$, and an effective mAS of 80 .

For this study, the CT images were transferred to a workstation (Syngo.via; Siemens, Germany) and evaluated by 2 radiologists (A.G.E. and G.D.) with 4 and 11 years of experience, respectively. The diameters of the irradiated and nonirradiated IMAs were measured in the first and second intercostal spaces (Fig. 2a, b). Three consecutive measurements were performed on axial images by each radiologist, and the mean measurement was noted. Luminal diameters were measured with intraluminal contrast material-enhanced imaging. IMAs in the third intercostal space could not be accurately measured because of their extremely thin diameters. The presence of calcification and any variation on both sides of the IMA were also reported.

\section{Statistical Analysis}

Data obtained in the study were analyzed using SPSS v23.0 (SPSS Inc., Chicago, IL, USA). Descriptive statistics for quantitative variables were given as means \pm standard deviation for quantitative variables and $n$ (\%) for qualitative variables. The independent-samples $t$ test was performed to compare the means of 2 groups. Correlations between 2 variables were examined using the Spearman rank-order correlation coefficient. $p<0.05$ was considered significant. 


\section{Results}

The mean age of the patients was $51 \pm 10$ years. The number of patients with left and right breast cancer was 131 (55\%) and 108 (45\%), respectively. The prescribed RT dose to the unilateral IMA was 46 Gy in 1 (0.5\%), 50 Gy in 227 (95\%), 56 Gy (with a 2.24-Gy fractionated dose) in 6 (2.5\%), and $60 \mathrm{~Gy}$ (with $2 \mathrm{~Gy} /$ fraction) in $5(2 \%)$ patients, respectively. The median of the mean and maximum dose to the IMA was $50 \mathrm{~Gy}$ (range 45-61 Gy) and 52 Gy (48-65 Gy), respectively. The RT technique was 3D-CRT in 23 (9.6\%), FP-IMRT in $82(34.3 \%)$, and IP-IMRT in 134 (56.1\%) patients, respectively.

There was no anatomical variation detected in the IMA in any patients. The mean diameter of the irradiated IMAs was $2.39 \pm 0.50 \mathrm{~mm}$ and $1.77 \pm 0.46 \mathrm{~mm}$ in the first and second intercostal spaces, respectively. The corresponding mean diameter of the nonirradiated IMAs was $2.67 \pm 0.49 \mathrm{~mm}(p<0.001)$ and $2.11 \pm 0.42 \mathrm{~mm}(p<$ 0.001 ), respectively (Fig. 3a, b). The diameters of the IMA were $<1.5 \mathrm{~mm}$ in $14(5.8 \%)$ patients in the first intercostal space, and in $69(28.8 \%)$ patients in the second intercostal space, respectively.

When the laterality was compared, the mean diameter of the irradiated IMAs in the first intercostal space was $2.49 \pm 0.48 \mathrm{~mm}$ and $2.31 \pm 0.50 \mathrm{~mm}$ on the right and left sides, respectively. The corresponding values of the nonirradiated IMAs were $2.62 \pm 0.49 \mathrm{~mm}$ and $2.70 \pm 0.49$ $\mathrm{mm}$, respectively. In the second intercostal space, the mean diameter of the irradiated IMAs was $1.80 \pm 0.40$ $\mathrm{mm}$ and $1.60 \pm 0.40 \mathrm{~mm}$ on the right and left sides, respectively. The corresponding values of the non-irradiated IMAs were $2.0 \pm 0.30 \mathrm{~mm}$ and $2.10 \pm 0.40 \mathrm{~mm}$, respectively. The diameters of the irradiated IMAs were significantly reduced compared to those of the nonirradiated IMAs. Figure 4 shows the difference (with $p$ values).

To add the effect of possible variations, the diameters of the nonirradiated IMAs of both sides were compared. The diameter of the nonirradiated left-side IMA was determined to be wider than that of the right-side IMA in both the first and second intercostal spaces, but without statistical significance $(p=0.2$ and $p=0.3$ in the first and second intercostal space, respectively). Calcification in the IMA was reported in $39(16.3 \%)$ patients and all of these were detected on the irradiated side.

Boost RT dose was not correlated to the vessel diameter or calcification. No statistically significant difference was found in the first or second intercostal space, respectively ( $p=0.1$ and $p=0.5$, respectively). There was no statistically significant difference between the boost dose and calcification either $(p=0.4)$.

The effect of the RT technique on vessel diameters and the presence of calcification were also evaluated. No sta-

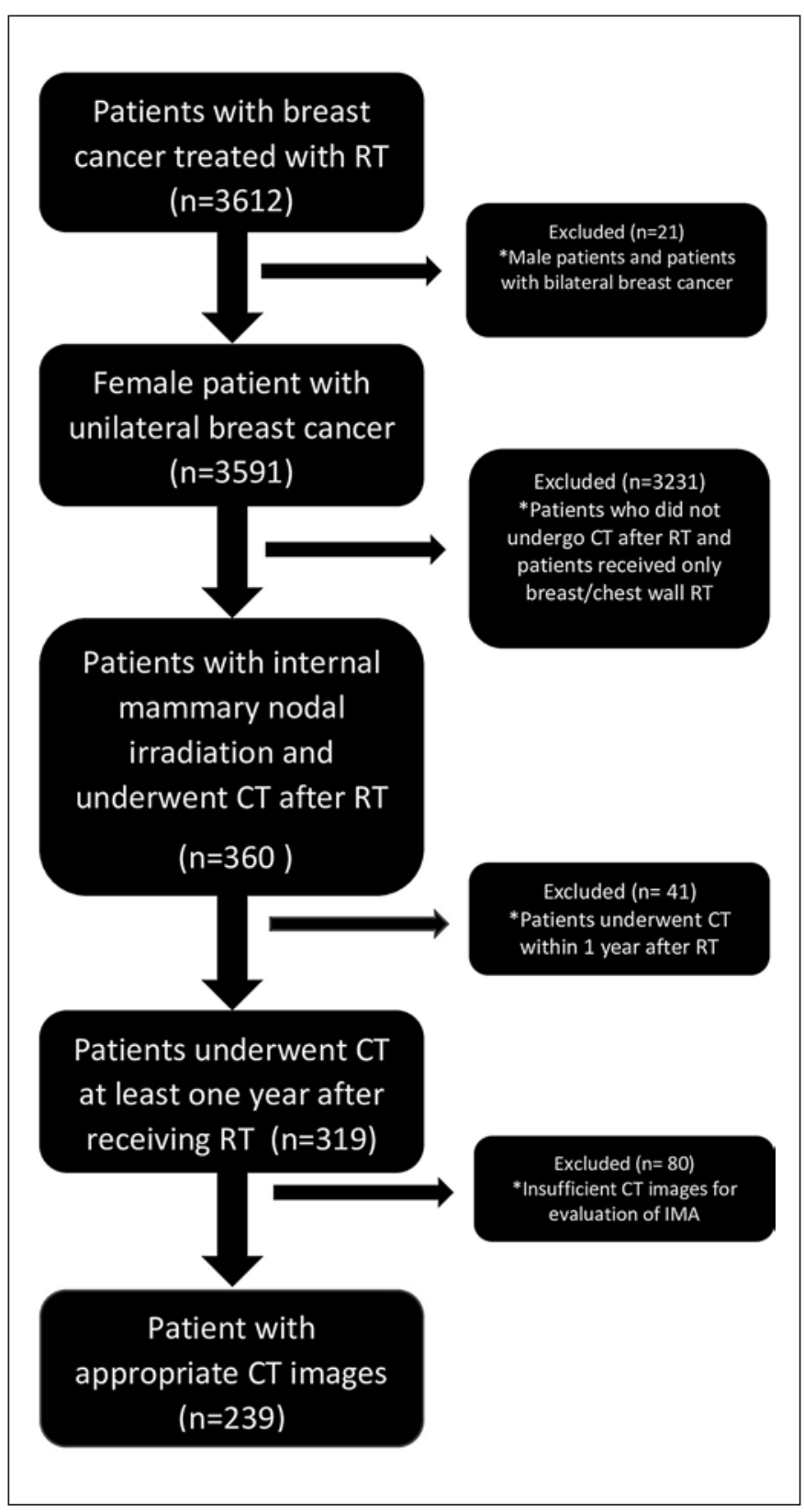

Fig. 1. Flow chart showing how cases were selected.

tistically significant difference was found in the first ( $p=$ $0.9)$ and second $(p=0.8)$ intercostal spaces, respectively, when 3D-CRT, FP-IMRT, or IP-IMRT was administered. There was no statistical significance between calcification and RT technique either $(p=0.4)$.

The median time interval between RT and CT scan was $22 \pm 15.1$ (range 12-69) months. The presence of calcification tended to appear in patients with a longer time interval between RT and CT $(p=0.6)$. The time interval between RT and CT did not correlate with the IMA diameter $(p=0.1$ and $p=0.08$ for the first and second intercostal space, respectively). 
Fig. 2. a Dose color wash demonstrates the region exposed to radiation. While the ipsilateral internal mammary node (IMN) is within the radiation zone, the contralateral IMN is outside the radiation zone (red circles). b Contrast-enhanced CT showing the locations of ipsilateral and contralateral internal mammary arteries (red circles).
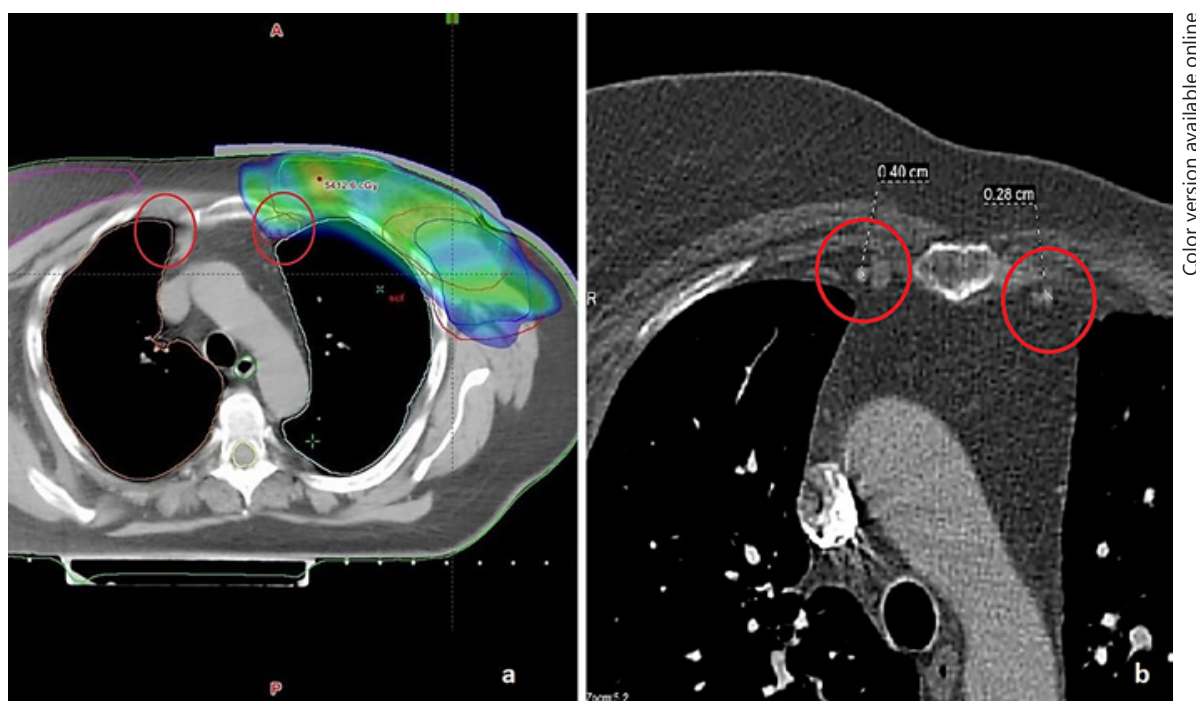

Fig. 3. Contrast-enhanced CT showing stenosis of the internal mammary artery in axial (a) and coronal (b) images. The scar resulting from lumpectomy surgery is indicated by the black star.
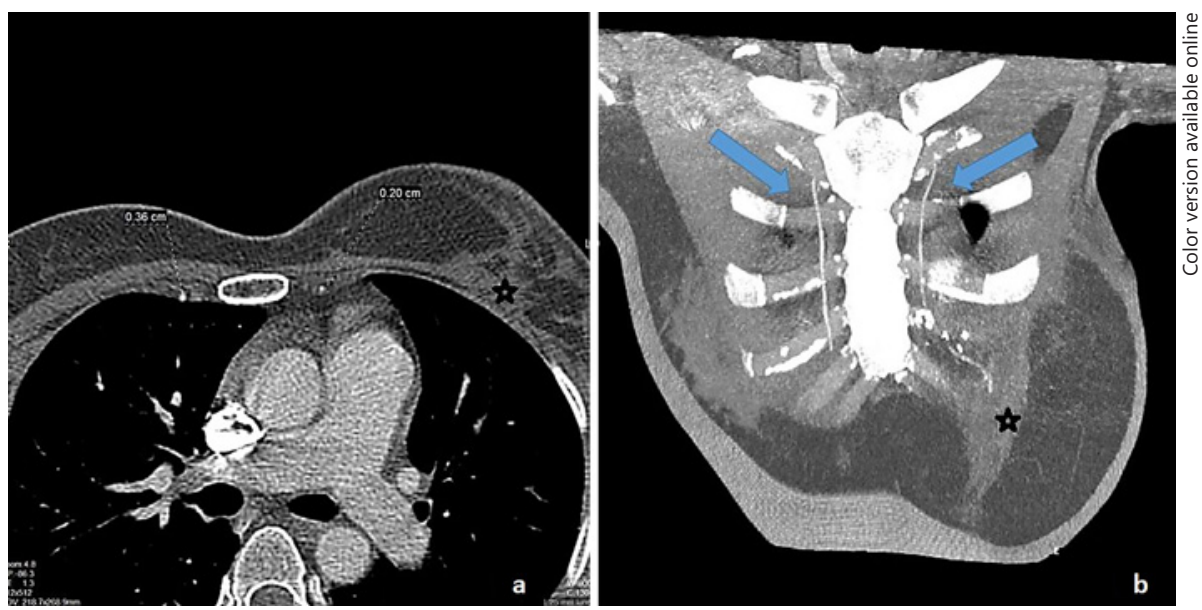

\section{Discussion}

The stenosis of vessels is often caused by the development of atherosclerotic plaques. The degree of stenosis can be assessed by measuring the luminal diameter of the vessel, not only on catheter-based angiography (the gold standard in this area for many years) but also on CT angiography (CTA) [15]. In this study, we tried to determine the effect of ionizing radiation on the IMA. On CTA, with an intraluminal contrast material, we measured the IMA luminal diameter as a radiological sign of atherosclerosis. We also evaluated the CT images for arterial calcifications of IMA. The major finding was that the diameter of the IMA was decreased due to RT regardless of the affected side. The second finding was that radiation induced calcification. The patency of the IMA is important in breast cancer cases for 2 reasons: (1) the IMA is an excellent conduit for CABG, and (2) it is the most com- mon recipient vessel used in free-flap breast reconstruction after mastectomy [7-9].

Two recent trials, the European Organization for Research and Treatment of Cancer (EORTC) 22922 and the MA.20, reported that adding regional nodal irradiation (RNI) to whole-breast or chest-wall RT after surgery improves locoregional control and decreases the rate of distant metastases $[3,4]$. However, RNI including IMNs may increase the risk of long-term cardiac toxicity in patients with left-sided breast cancer via micro- and macrovascular injuries [16]. In these patients, the left anterior descending (LAD) coronary artery receives the highest radiation doses, which causes stenosis via vascular endothelial damage and intimal proliferation. As a result, coronary artery disease (CAD) is observed, which is characterized as radiation-induced atherosclerotic plaque formation [9]. Although increased risk of cardiac toxicity starts within the first 5 years after RT and continues there- 


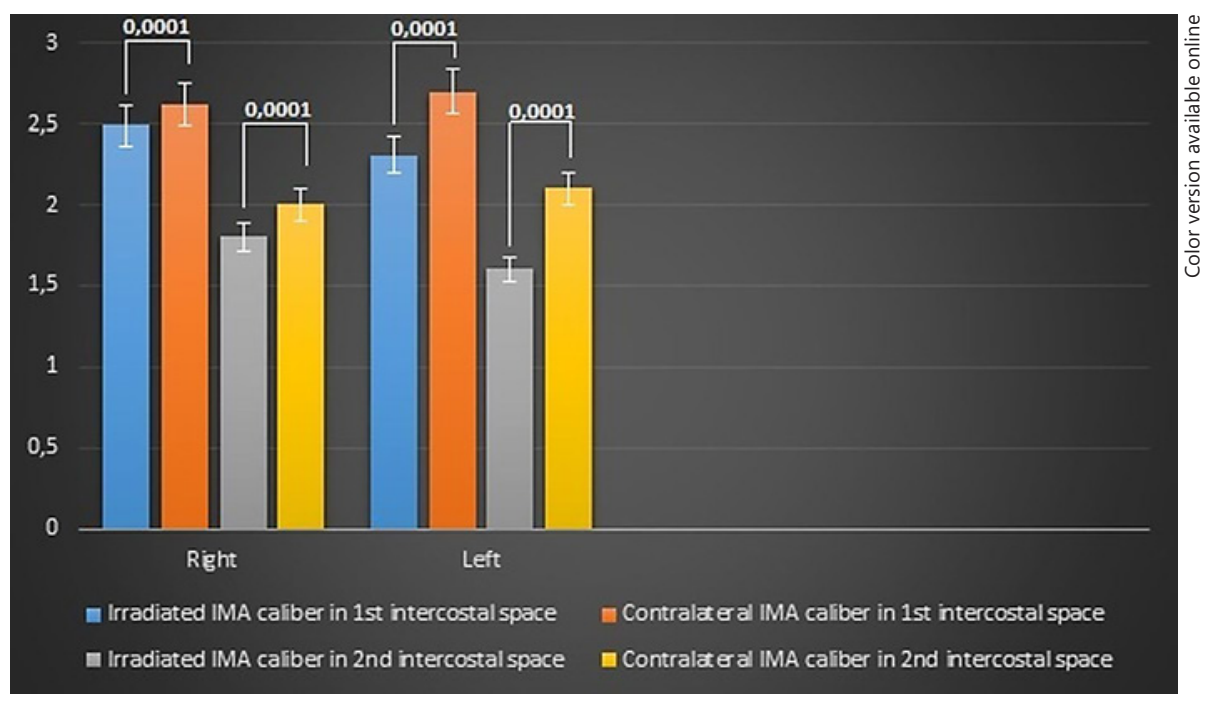

Fig. 4. Comparisons of mean measurements of the irradiated internal mammary artery (IMA) and nonirradiated contralateral IMA are shown for both right and left sides. The statistical significance level is given between the bars for each comparison.

after, high-dose exposure ( $>30 \mathrm{~Gy}$ ) causes cardiac complications that become apparent within 1-2 years of exposure $[17,18]$. In order to observe the late effects of radiation on the IMA radiologically, only the patients who had a CT evaluation $>1$ year after RT were included in our study.

in a population-based case-control study, Darby et al. [19] demonstrated that with every 1-Gy increase in mean radiation dose, there was a $7.4 \%$ relative increased risk of major coronary events with no apparent threshold. CAD initially tends to be asymptomatic, but it may present later with stable or unstable angina pectoris, sudden cardiac death, or acute coronary syndrome. In addition to RT, chemotherapy, especially with anthracyclines, increases the risk of heart disease in breast cancer patients [7-9]. Radiation-induced CAD may require CABG during follow-up and display worse outcomes when compared to the general population.

Irradiation of the IMNs can cause stenosis, not only of the coronary arteries but also the IMA which is located next to the IMNs. Similarly, in our study, the mean luminal diameter of the IMA was found to be decreased due to RT, regardless of the affected side. This may cause difficulties for future CABG which may be required in patients with CAD secondary to RT. A previous study, investigating the effect of radiation on the IMA via transthoracic Doppler echocardiography probe intraoperatively, showed no significant difference between irradiated and nonirradiated individuals [20]. The mean interval between RT and cardiac surgery was 395 days and there was no difference in the clinical outcome and mortality at 18 months. In contrast, Brown et al. [21] found that IMA patency after CABG in patients with prior mediastinal RT was lower than anticipated for patients without RT. The patency of the IMA, the most commonly used conduit for CABG, is therefore extremely important in left-sided breast cancer patients who are more likely to develop CAD than the general population. Evaluation of the diameter of the IMA by CT prior to CABG may be helpful in these cases.

Another finding was that the RT technique or the administration of a boost dose did not affect the vessel diameter. The reason for the lack of statistical significance could have been the relatively small sample size, particularly of those patients that received a boost dose and underwent the 3D-CRT technique. The other possible reason is that all our patients received at least 46 Gy of RT which is far beyond the threshold dose the lower dose that spares the vessel from damage.

The time interval between RT and CT was found to not be correlated with the vessel diameter. Previous studies have shown that RT causes endothelial dysfunction and inflammation for up to 6 months after exposure [22]. In this study, only patients that underwent CT at least 1 year after RT were included so it is possible that the stationary chronic effects of RT were observed.

As another strong indicator of atherosclerotic disease, vascular calcification can be detected by noninvasive imaging modalities such as CT $[23,24]$. The presence of IMA calcification was also evaluated in our study and was detected in only $16.3 \%$ of patients, all on the irradiated side. Such calcifications detected on follow-up CT in breast cancer survivors have rarely been reported after RT [25]. Several factors (diabetes, hypertension, hyperlipidemia, chronic renal failure, etc.) play a role in the development of vascular calcifications [23]. However, as no calcifications were observed in the contralateral IMA, we assume that the calcifications suggested a sequela of radiation injury. Calcification tended to appear in patients with a longer time interval between RT and CT, but it did 
not reach statistical significance $(p=0.6)$. We did not find any correlation between boost dose or RT technique and calcification. The reasons for this may have been the relatively small sample size and an increased fibrous noncalcified component in the atherosclerotic plaques of patients that underwent RT.

Laterality was evaluated separately because of a possible difference between the right and left sides. A previous study investigating the IMA via CTA in 110 female patients who did not undergo RT revealed wider diameters on the right side [26], but in our study the diameters were wider on the left, nonirradiated side (without statistical significance). However, the irradiated right-side IMAs had a larger mean diameter than the irradiated left-side IMAs.

Another important role of the IMA in breast cancer patients is in breast reconstruction. After a mastectomy, breast reconstruction provides psychosocial benefits regarding body image, self-esteem, sexuality, and quality of life [27]. Largely supported by the literature, it has been found that, in comparison with implantbased reconstruction, patients with autologous reconstruction had greater esthetic satisfaction, overall longterm satisfaction, and required fewer clinic visits [2830]. In addition, it is the only way to achieve breast reconstruction after $\mathrm{RT}$ to the chest wall. Therefore, as the most common recipient vessel used in free-flap breast reconstruction, the IMA is of major importance for breast cancer patients.

Leppard et al. [31] reported that the IMA can be used as the primary recipient vessel in autologous breast reconstruction even in case of previous irradiation, based on their retrospective analysis in 154 patients. In their study, the mean internal mammary vein diameter was reported as $2.8 \mathrm{~mm}$. In contrast, the IMA diameter was $<1.5 \mathrm{~mm}$ in 5.8 and $28.8 \%$ of the patients in the first and second intercostal space, respectively. Though there are no data on the effect on flap failure of a reduced IMA diameter after RT, it is common knowledge that the smaller the vessel diameter, the higher the rate of flap failure [32]. Analysis of the IMA via CTA prior to surgery can serve as an invaluable tool for surgeons performing microvascular procedures, and flap complications can be avoided.

Our results may be more valuable for the evaluation of the long-term effects of radiation on the IMA. To the best of our knowledge, this is the first study that shows the effects of IMN irradiation on the IMA via contrast-enhanced thorax CT. The main advantage in our study was that the nonirradiated side was used as the control; this enabled us to make a conclusion about the radiation effects alone on the IMA, i.e., regardless of other common atherosclerotic risk factors (e.g., diabetes, hypercholesterolemia, hypertension, obesity, and cigarette smoking).
However, there are some limitations to our study. The major limitation is its retrospective nature. Although significant differences were determined in vessel diameters, the relationship between vessel diameter and flap failure could not be evaluated. There was also no information about ischemic cardiac events in patients with irradiated IMAs who underwent CABG. In addition, the evaluation was performed in only 1 follow-up thorax CT, so we could not assess the change over time in the IMAs. As another limitation, the presence of common atherosclerotic risk factors in patients was not known. Finally, a slight physiologic difference between the diameters of the right and left IMAs may exist in the general population. However, according to our results, although the diameter of the left IMA was a little wider than that of the right IMA on the nonirradiated side, this did not reach statistical significance, and the IMA diameters were decreased due to RT regardless of the side.

\section{Conclusion}

The diameter of the IMA in breast cancer patients decreased due to RT regardless of the laterality, RT technique, or administration of a boost dose. Evaluation of vessels on CT before CABG and flap reconstruction can help the surgeon select the most appropriate vessel.

\section{Statement of Ethics}

Study approval was granted by the institutional ethics board. Written informed consent is not applicable due to retrospective nature of the study.

\section{Disclosure Statement}

The authors have no conflicts of interest to declare.

\section{Funding Sources}

The authors received no financial support.

\section{Author Contributions}

All authors contributed to the study conception and design, preparation of the material, and the collection, analysis, and interpretation of data. The first draft of the manuscript was written by G.D., A.G.E., S.Y.S., M.G., and J.K. It was critically revised for important intellectual content by M.G.A., G.Ö., F.Y., and F.D. All authors read and approved the final manuscript and agreed to be accountable for all aspects of the work. 


\section{References}

1 Veronesi U, Marubini E, Mariani L, Valagussa P, Zucali R. The dissection of internal mammary nodes does not improve the survival of breast cancer patients. 30-year results of a randomised trial. Eur J Cancer. 1999 Sep; 35(9):1320-5.

2 Huang O, Wang L, Shen K, Lin H, Hu Z, Liu $\mathrm{G}$, et al. Breast cancer subpopulation with high risk of internal mammary lymph nodes metastasis: analysis of 2,269 Chinese breast cancer patients treated with extended radical mastectomy. Breast Cancer Res Treat. 2008 Feb;107(3):379-87.

3 Poortmans PM, Collette S, Kirkove C, Van Limbergen E, Budach V, Struikmans H, et al.; EORTC Radiation Oncology and Breast Cancer Groups. Internal Mammary and Medial Supraclavicular Irradiation in Breast Cancer. N Engl J Med. 2015 Jul;373(4):317-27.

4 Whelan TJ, Olivotto IA, Parulekar WR, Ackerman I, Chua BH, Nabid A, et al.; MA.20 Study Investigators. Regional Nodal Irradiation in Early-Stage Breast Cancer. N Engl J Med. 2015 Jul;373(4):307-16.

5 Yusuf SW, Sami S, Daher IN. Radiation-induced heart disease: a clinical update. Cardiol Res Pract. 2011 Feb;2011:317659.

6 Linton MF, Babaev VR, Huang J, Linton EF Tao H, Yancey PG. Macrophage Apoptosis and Efferocytosis in the Pathogenesis of Atherosclerosis. Circ J. 2016 Oct;80(11):2259-68.

7 Nilsson G, Holmberg L, Garmo H, Duvernoy O, Sjögren I, Lagerqvist B, et al. Distribution of coronary artery stenosis after radiation for breast cancer. J Clin Oncol. 2012 Feb;30(4): 380-6.

8 van der Pal HJ, van Dalen EC, van Delden E, van Dijk IW, Kok WE, Geskus RB, et al. High risk of symptomatic cardiac events in childhood cancer survivors. J Clin Oncol. 2012 May;30(13):1429-37.

9 Raghunathan D, Khilji MI, Hassan SA, Yusuf SW. Radiation-Induced Cardiovascular Disease. Curr Atheroscler Rep. 2017 May;19(5): 22.

10 Temple CL, Strom EA, Youssef A, Langstein HN. Choice of recipient vessels in delayed TRAM flap breast reconstruction after radiotherapy. Plast Reconstr Surg. 2005 Jan;115(1): 105-13.

11 Clark CP 3rd, Rohrich RJ, Copit S, Pittman $\mathrm{CE}$, Robinson J. An anatomic study of the internal mammary veins: clinical implications for free-tissue-transfer breast reconstruction. Plast Reconstr Surg. 1997 Feb;99(2):400-4.
12 Nahabedian M. The internal mammary artery and vein as recipient vessels for microvascular breast reconstruction. Ann Plast Surg. 2012 May;68(5):537-8.

13 Galanis C, Nguyen P, Koh J, Roostaeian J, Festekjian J, Crisera C. Microvascular lifeboats: a stepwise approach to intraoperative venous congestion in DIEP flap breast reconstruction. Plast Reconstr Surg. 2014 Jul;134(1):207.

14 White J, Tai A, Arthur D, Buchholz T, MacDonald S, Mark L, et al; RTOG Collaborators. [Internet] Breast Cancer Atlas for Radiation Therapy Planning: Consensus Definitions. Available from: https://www.rtog.org/LinkClick.aspx?fileticket $=$ vzJFhPaBipE $=$

15 Ota H, Takase K, Rikimaru H, Tsuboi M, Yamada T, Sato A, et al. Quantitative vascular measurements in arterial occlusive disease. Radiographics. 2005 Sep-Oct;25(5):1141-58.

16 Gagliardi G, Constine LS, Moiseenko V, Correa C, Pierce LJ, Allen AM, et al. Radiation dose-volume effects in the heart. Int J Radiat Oncol Biol Phys. 2010 Mar;76(3 Suppl): S77-85.

17 Heidenreich PA, Schnittger I, Strauss HW, Vagelos RH, Lee BK, Mariscal CS, et al. Screening for coronary artery disease after mediastinal irradiation for Hodgkin's disease. J Clin Oncol. 2007 Jan;25(1):43-9.

18 Darby SC, Cutter DJ, Boerma M, Constine LS, Fajardo LF, Kodama K, et al. Radiation-related heart disease: current knowledge and future prospects. Int J Radiat Oncol Biol Phys. 2010 Mar;76(3):656-65.

19 Darby SC, Ewertz M, McGale P, Bennet AM, Blom-Goldman U, Brønnum D, et al. Risk of ischemic heart disease in women after radiotherapy for breast cancer. N Engl J Med. 2013 Mar;368(11):987-98.

20 Nasso G, Canosa C, De Filippo CM, Modugno P, Anselmi A, Gaudino M, et al. Thoracic radiation therapy and suitability of internal thoracic arteries for myocardial revascularization. Chest. 2005 Sep;128(3):1587-92.

21 Brown ML, Schaff HV, Sundt TM. Conduit choice for coronary artery bypass grafting after mediastinal radiation. J Thorac Cardiovasc Surg. 2008 Nov;136(5):1167-71.

22 Tungjai M, Whorton EB, Rithidech KN. Persistence of apoptosis and inflammatory responses in the heart and bone marrow of mice following whole-body exposure to ${ }^{28}$ Silicon $\left({ }^{28} \mathrm{Si}\right)$ ions. Radiat Environ Biophys. 2013 Aug;52(3):339-50
23 Farzaneh-Far A, Proudfoot D, Shanahan C, Weissberg PL. Vascular and valvar calcification: recent advances. Heart. 2001 Jan;85(1): 13-7.

24 Schoenhagen P, Halliburton SS, Stillman AE, Kuzmiak SA, Nissen SE, Tuzcu EM, et al. Noninvasive imaging of coronary arteries: current and future role of multi-detector row CT. Radiology. 2004 Jul;232(1):7-17.

25 Takx RA, Vliegenthart R, Schoepf UJ, Pilz LR, Schoenberg SO, Morris PB, et al. Coronary artery calcium in breast cancer survivors after radiation therapy. Int J Cardiovasc Imaging. 2017 Sep;33(9):1425-31.

26 Cook JA, Tholpady SS, Momeni A, Chu MW Predictors of internal mammary vessel diameter: A computed tomographic angiographyassisted anatomic analysis. J Plast Reconstr Aesthet Surg. 2016 Oct;69(10):1340-8.

27 Albornoz CR, Bach PB, Mehrara BJ, Disa JJ, Pusic AL, McCarthy CM, et al. A paradigm shift in U.S. Breast reconstruction: increasing implant rates. Plast Reconstr Surg. 2013 Jan; 131(1):15-23.

28 Visser NJ, Damen TH, Timman R, Hofer SO, Mureau MA. Surgical results, aesthetic outcome, and patient satisfaction after microsurgical autologous breast reconstruction following failed implant reconstruction. Plast Reconstr Surg. 2010 Jul;126(1): 26-36.

29 Gart MS, Smetona JT, Hanwright PJ, Fine NA, Bethke KP, Khan SA, et al. Autologous options for postmastectomy breast reconstruction: a comparison of outcomes based on the American College of Surgeons National Surgical Quality Improvement Program. J Am Coll Surg. 2013 Feb;216(2):229-38.

30 Pusic AL, Matros E, Fine N, Buchel E, Gordillo GM, Hamill JB, et al. Patient-Reported Outcomes 1 Year After Immediate Breast Reconstruction: Results of the Mastectomy Reconstruction Outcomes Consortium Study. J Clin Oncol. 2017 Aug;35(22):2499506.

31 Leppard W, Pomposelli T, Chang EI, Suliman A, Herrera F. Internal mammary usability as recipient vessels in DIEP breast reconstruction in the setting of previous radiation. J Plast Reconstr Aesthet Surg. 2018 Aug;71(8):1123-

32 Feng LJ. Recipient vessels in free-flap breast reconstruction: a study of the internal mammary and thoracodorsal vessels. Plast Reconstr Surg. 1997 Feb;99(2):405-16. 